\title{
Primary Malignant Melanoma of the Larynx: A Case Report
}

\author{
Rajlaxmi Panigrahi ${ }^{1}$, Soumya Jee ${ }^{2}$
}

\begin{abstract}
Malignant melanoma of the larynx is a rare cancer that can appear as a primary tumor or as a metastasis from a cutaneous head and neck primary site. It is known for its poor outcomes and survival. Owing to rarity of the disease every reported case of primary malignant melanoma of the larynx is important for understanding the natural course of disease, presentation, tumor biology, treatment, and prognosis for establishing guidelines for further management of the disease. Here we report a case of primary malignant melanoma of the larynx. Diagnosis was based on histological, immunohistochemical findings from excisional biopsy specimen from the larynx.

Keywords: Larynx, Malignant, Melanoma, Primary, Tracheostomy.

International Journal of Phonosurgery \& Laryngology (2019): 10.5005/jp-journals-10023-1175
\end{abstract}

\section{INTRODUCTION}

Primary noncutaneous malignant melanomas of the head and neck is an aggressive and uncommon neoplasm. Malignant melanoma of larynx is a rare cancer that can appear as a primary tumor or as a metastasis from a cutaneous head and neck primary site. It is known for its poor outcomes and survival.

These account for $6-20 \%$ of head and neck malignant melanoma and about $1 \%$ of all melanoma cases. ${ }^{1,2}$ The most common sites for noncutaneous malignant melanoma of the head and neck are the sinonasal (55\%) and oral cavity (40\%). ${ }^{3}$

In view of rare incidence, every reported case of primary malignant melanoma of the larynx adds to the better understanding of disease presentation, spread, the treatment protocol, and the prognostic factor for establishing guidelines for further management of the tumor. So here we present a case of primary malignant melanoma of the larynx.

\section{Case Description}

An 82-year-old male presented to the outpatient department with difficulty in swallowing solids for 6 months that was gradually increasing in nature. He had hoarseness that was gradually progressing over last 6 months and difficulty in breathing for 4 days. There was no history of weight loss, fever, oral bleeding, or any other associated comorbidities such as diabetes or hypertension. There was no relevant family history. The patient was not addicted to alcohol and was a nonsmoker.

General physical examination and other systemic examination were within the normal limit. There was no palpable enlargement of neck nodes and on direct laryngoscopy there was polypoidal, proliferative, lobulated, pigmented growth seen in the larynx, involving the supraglottic region of larynx (Figs 1 and 2).

\section{Management}

The patient presented to us with hoarseness, dysphagia, and dyspnea. Tracheostomy was planned. Tracheostomy and biopsy were performed in the same sitting under general anesthesia.

Routine hemogram, blood biochemistry, and chest X-ray were within the normal limit. No evidence of any cutaneous skin lesion was found. Ultrasonography of the whole abdomen was performed to rule out any suspected visceral lesion.
1,2Department of Otolaryngology, Hi Tech Medical College and Hospital, Bhubaneswar, Odisha, India

Corresponding Author: Soumya Jee, Department of Otolaryngology, $\mathrm{Hi}$ Tech Medical College and Hospital, Bhubaneswar, Odisha, India, Phone: +91 9439500080, e-mail: soumyajee01@gmail.com

How to cite this article: Panigrahi $R$, Jee $S$. Primary Malignant Melanoma of the Larynx: A Case Report. Int J Phonosurg Laryngol 2019;9(2):47-48.

Source of support: HiTech Medical College and Hospital, Bhubaneswar, Odisha, India

Conflict of interest: None

Contrast enhanced computed tomography (CECT) neck is suggestive of the ill-defined asymmetric soft tissue thickening in the right posterior pharyngeal wall causing mild encroaching up to the pharyngeal lumen (? Neoplastic). Advised for biopsy. Direct laryngoscopy and debulking of mass was done under general anesthesia. The excised mass was sent for histopathological examination (Fig. 3).

Histomorphology revealed a polyp with an infiltrating malignant neoplasm in the submucosa. The tumor cells are round to oval to spindle with moderate nuclear pleomorphism

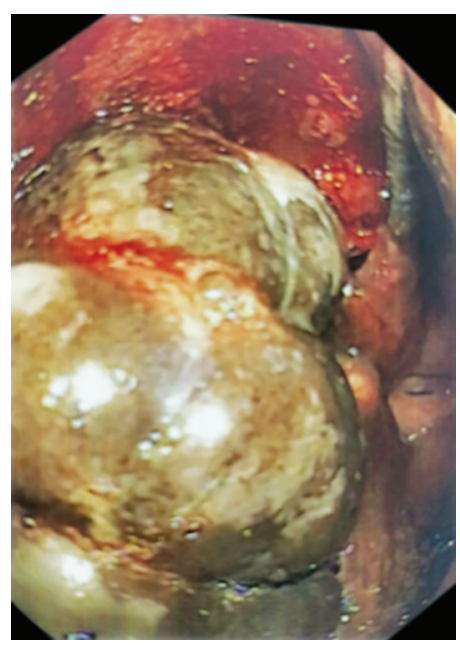

Fig. 1: Direct laryngoscopy showing lobulated pigmented growth

o The Author(s). 2019 Open Access This article is distributed under the terms of the Creative Commons Attribution 4.0 International License (https://creativecommons. org/licenses/by-nc/4.0/), which permits unrestricted use, distribution, and non-commercial reproduction in any medium, provided you give appropriate credit to the original author(s) and the source, provide a link to the Creative Commons license, and indicate if changes were made. The Creative Commons Public Domain Dedication waiver (http://creativecommons.org/publicdomain/zero/1.0/) applies to the data made available in this article, unless otherwise stated. 


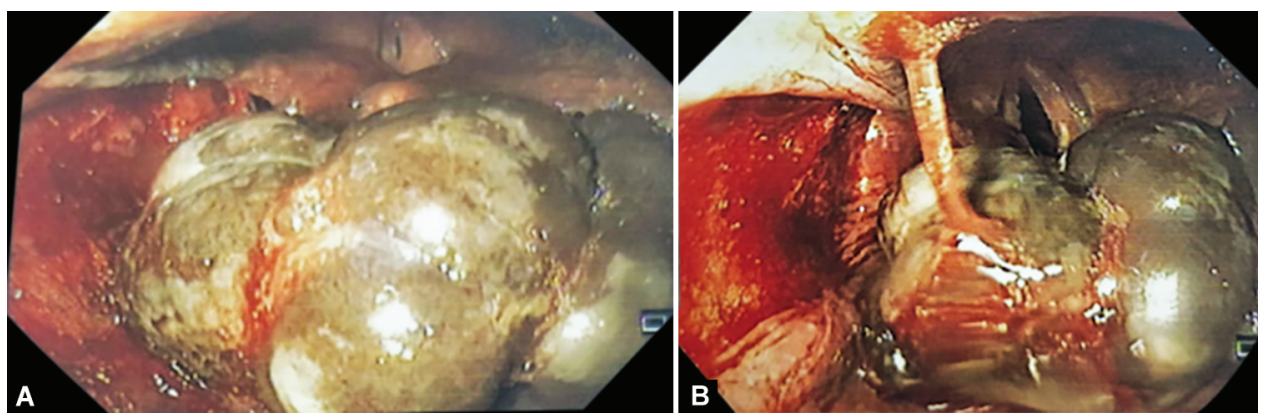

Figs $2 \mathrm{~A}$ and B: Polypoidal pigmented growth involving the supraglottic region of larynx

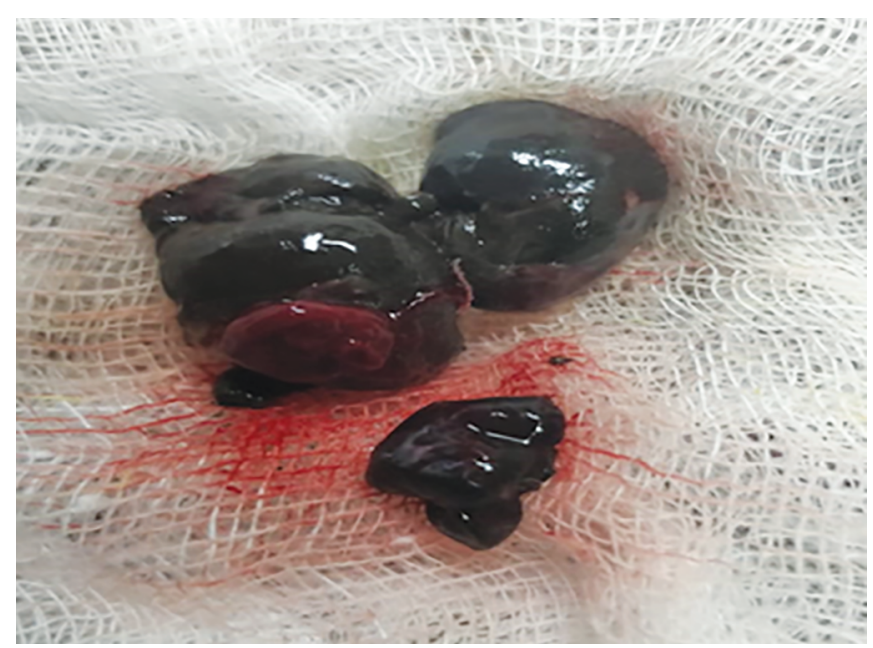

Fig. 3: Specimen sent for histopathological assessment

and cytoplasmic melanin pigment and nuclear predominance. Immunohistochemistry suggested immunoreactivity with diffuse positivity for Melan- $\mathrm{A}, \mathrm{S}-100$ protein, $\mathrm{HMB}-45$, and vimentin against a pleomorphic background of malignant melanoma.

Positron emission tomography/computed tomography (PET CT) done for pretherapy evaluation suggested abnormal fluorodeoxyglucose (FDG) concentration involving a primary mass lesion as tissue thickening in the posterior wall of the supraglottic larynx involving bilateral pyriform sinuses and aryepiglottic folds measuring $4 \times 1.8 \mathrm{~cm}$ with SUV MAX-15.18. No regional nodal or any visceral metastasis.

Given the fact that the patient is more than 80 years and total laryngectomy even if technically possible but perioperative complications will have significant morbidity at this age. Relatives were counseled about the role of radiotherapy in this condition, attempted as a palliative measure. Malignant melanoma of the head and neck is aggressive and carry a poor prognosis.

\section{Discussion}

Melanocytes are neuroectodermal in origin. Usually migrate to ectodermal and mucosal structures such as skin, uveal tract, retina, and other ectodermal structures. Very rarely they migrate to nasopharynx, larynx, or tracheobronchial mucosa. This explains the rarity of this disease in these locations.

The first case of noncutaneous melanoma was described in 1856 by Waber, ${ }^{4}$ but distinct entity was given by Luke in $1869 .{ }^{5}$ In the past, no clear distinction regarding the origin as primary or metastatic was made in the literature. Primary melanomas are very rare neoplasms and are diagnosis of exclusion against a background of positive histopathology. The etiopathogenesis of primary mucosal melanomas has not yet been fully elucidated. The growth pattern of primary mucosal melanoma of the larynx in our case resembles a polypoidal pattern. Classification according to depth (Breslow) is not suitable for these kind of locally advanced disease at presentation. The tumor, node, metastasis (TNM) system is advocated for most head and neck cancer including melanomas. ${ }^{6}$ No T1-2 or stage I and stage II was included in American Joint Committee on Cancer (AJCC) as mucosal noncutaneous melanomas are aggressive tumors in the head and neck. Because of their aggressive biology, prognosis still remains grave despite adjuvant treatment. Older mean age of presentation and associated age-related comorbidities concern radical surgery. Because of the rarity of primary laryngeal melanomas, every reported case is important for understanding the natural course of disease, presentation, tumor biology treatment, and prognosis for establishing guidelines for further management of the disease.

\section{References}

1. Amin $\mathrm{HH}$, Petruzzelli GJ, Husain A, et al. Primary malignant melanoma of larynx. Aron Pathol Lab Med 2001;25(2):271-273. DOI: 10.1043/0003-9985(2001)1252.0.CO;2.

2. Patel SG, Prasad ML, Escrig M, et al. Primary mucosal malignant melanoma of the head and neck. Head Neck 2002;24(3):247-257. DOI: $10.1002 /$ hed.10019.

3. Seetharamu N, Ott PA, Paviick AC. Mucosal melanoma: a case based review of the literature. Oncologist 2010;15(7):772-773. DOI: 10.1634/ theoncologist.2010-0067.

4. Weber CO. Chirurgische Ehrfahrungen, nebst zahlreichen Beobachtungen aus der Chirurgischen Klinik. Berlin, Germany. Reimer 1859.

5. Luke A Die Lehre vin den Geschwulsten in anatomischer Klinischer Beziehung in Handuch d.allg u.spec chir Erlangen. 1869:244.

6. Ballantyne AJ. Malignant melanoma of the skin and head and neck. Am J Surg 1970;120(4):425-431. DOI: 10.1016/s0002-9610(70)80001-0. 